\title{
Cost-effectiveness analysis of genotype screening and therapeutic drug monitoring in patients with inflammatory bowel disease treated with azathioprine therapy: a Chinese healthcare perspective using real-world data
}

\author{
Dayong Zeng", Xiaoting Huang", Shen Lin, Rongfang Lin, Xiuhua Weng, Pinfang Huang \\ Department of Pharmacy, The First Affiliated Hospital of Fujian Medical University, Fuzhou, China \\ Contributions: (I) Conception and design: D Zeng, X Huang; (II) Administrative support: S Lin; (III) Provision of study materials or patients: R Lin; (IV) \\ Collection and assembly of data: D Zeng, X Huang, S Lin, R Lin; (V) Data analysis and interpretation: D Zeng, X Huang; (VI) Manuscript writing: \\ All authors; (VII) Final approval of manuscript: All authors. \\ \#These authors contributed equally to this work. \\ Correspondence to: Xiuhua Weng; Pinfang Huang. Department of Pharmacy, The First Affiliated Hospital of Fujian Medical University, No. 20 Cha \\ Zhong Road, Taijiang, Fuzhou, China. Email: wxh001@fjmu.edu.cn; abstract2016@163.com.
}

Background: This study aimed to analyze the cost-effectiveness of combining screening for thiopurine methyl transferase (TPMT) and nucleotide triphosphate diphosphatase (NUDT15) defective alleles with therapeutic drug monitoring (TDM) in Chinese patients with inflammatory bowel disease (IBD) treated with azathioprine (AZA).

Methods: We evaluated the cost-effectiveness of combining screening for NUDT15 and TPMT deficiency with TDM in patients receiving AZA treatment over a 1-year horizon by developing a decision tree model. Real-world data and published literature were used to derive model inputs. The model's primary outcomes included quality-adjusted life-years (QALYs) and incremental cost-effectiveness ratios (ICERs). One-way and probabilistic sensitivity analyses were used to address uncertainty.

Results: Compared to NUDT15 genotyping, the combined TPMT/NUDT15 genotyping strategy cost an additional \$13.83, yielding an ICER of \$3,929.54/QALY, which was under the willingness-to-pay level of \$30,425 per QALY in China. Compared to strategies with singular TPMT genotyping or no genotyping, the combined TPMT/NUDT15 genotyping strategy gained 0.00406 and 0.00782 QALYs and reduced the cost by $\$ 25.15$ and $\$ 99.06$, respectively. Additionally, incorporating TDM of AZA was more effective and less expensive than strategies without TDM. One-way sensitivity analysis revealed the expense attached to severe myelotoxicity to be the factor with the greatest influence in the present research. The application of the combined genotype screening strategy with TDM of AZA treatment was found to have a $91.7 \%$ chance of being cost-effective.

Conclusions: For Chinese patients with IBD who receive an AZA regimen, a strategy involving combined NUDT15/TPMT genotype screening prior to treatment initiation and incorporating TDM for treatment management is cost-effective compared to strategies involving genotyping of NUDT15 or TPMT alone or genotyping without TDM.

Keywords: Genotype screening; therapeutic drug monitoring (TDM); inflammatory bowel disease (IBD); azathioprine (AZA); cost-effectiveness

Submitted Feb 22, 2021. Accepted for publication Jul 07, 2021.

doi: $10.21037 / \mathrm{atm}-21-1980$

View this article at: https://dx.doi.org/10.21037/atm-21-1980

(c) Annals of Translational Medicine. All rights reserved. 


\section{Introduction}

Inflammatory bowel disease (IBD), which includes Crohn's disease and ulcerative colitis, has the main characteristic of chronic and idiopathic colorectal tract inflammation. According to how severe the IBD is and how it manifests clinically, therapies usually include mesalazine, immunosuppressive, corticosteroid, and biologic agents (1). Azathioprine (AZA) is an extraordinarily important immunosuppressive therapy aimed at maintaining the remission of IBD at an affordable cost to the patients (2). However, AZA unfortunately has significant adverse drug reactions (ADRs), with approximately $20 \%$ of patients developing ADRs, leading to treatment withdrawal within 2 years $(3,4)$. In particular, severe myelotoxicity leads to leukopenia and may even result in death.

Many ADRs of AZA are associated with polymorphic variants of the thiopurine methyl transferase (TPMT) gene (5). Consequently, regulatory agencies, including the United States Food and Drug Administration, recommend that TPMT gene testing is performed before AZA treatment is initiated (6). However, the clinical value of TPMT testing for predicting thiopurine-induced leukopenia remains controversial in Asian populations due to TPMT variants being less common in individuals of Asian race than in Caucasians (approximately $3 \%$ vs. $10 \%)$. Moreover, in China, the prevalence of TPMT variants is as low as $1.4 \%$ (7). Studies in patients with Asian ancestry have also identified variants within nucleotide triphosphate diphosphatase (NUDT15) as risk factors for thiopurine-induced leukopenia $(8,9)$. Currently, consensus guidelines and professional societies recommend that NUDT15 genotyping is performed to guide AZA treatment in Asian patients (10).

Compared with empirical dosage escalation or drugswitching strategies, the inclusion of therapeutic drug monitoring (TDM) in therapeutic management can improve the clinical outcome and safety for patients, as it optimizes drug and dosage selection and minimizes unreasonable drug use, thus decreasing medical costs (11). Due to the potentially harmful effects associated with reactive TDM of AZA therapy, including the additional burden of intensified laboratory monitoring for each dose adjustment and the potential delay to alternative effective therapies for patients who do not respond to AZA treatment, the American Gastroenterological Association advises against routine thiopurine metabolite monitoring. Therefore, incorporating TDM into treatment management strategies remains controversial (12).

In view of the high incidence of IBD in developed countries and the substantial increase in incidence in developing countries in recent years, IBD has evolved into a global burden. Genotype screening and incorporation of TDM into AZA treatment management strategies ultimately increase treatment expenditure, and thus raise the costeffectiveness of using TPMT and NUDT15 screening, and TDM in patients with IBD as an important matter to address.

Therefore, the present work sought to analyze whether a strategy that combined the screening of TPMT and NUDT15 genetic variants with TDM of AZA was costeffective in the prevention of severe thiopurine-induced leukopenia in the Chinese healthcare setting based on IBD epidemiology and real-world data. We present the following article in accordance with the CHEERS reporting checklist (13) (available at https://dx.doi.org/10.21037/atm21-1980).

\section{Methods}

\section{Model overview}

A decision-analytic model was adopted to determine the cost-effectiveness of a strategy incorporating combined genetic screening for NUDT15 and TPMT deficiency, and TDM for AZA treatment management from a Chinese healthcare perspective (14). The study participants were patients diagnosed with IBD by standard clinical, radiological, histological, and endoscopic assessment (15). Since most severe cases of AZA-induced myelotoxicity occur in the $1^{\text {st }}$ year after the initial treatment, a study horizon of 1 year was selected (16). Analyses were performed for the direct costs associated with genotype screening and TDM, and the costs of treatment. We supposed $100 \%$ patient compliance with the treatments recommended by test results. The study methods were in line with the recommendations of the CHEERS (13). All procedures performed in this study involving human participants were in accordance with the Declaration of Helsinki (as revised in 2013). The study was approved by the Ethics Committee of the First Affiliated Hospital of Fujian Medical University (approval number: 2015-131). All patients provided written informed consent before participation.

\section{Choice of model and description}

According to different possibilities, we evaluated the following 4 screening strategies: (I) genetic screening for TPMT; (II) genetic screening for NUDT15; (III) combined 


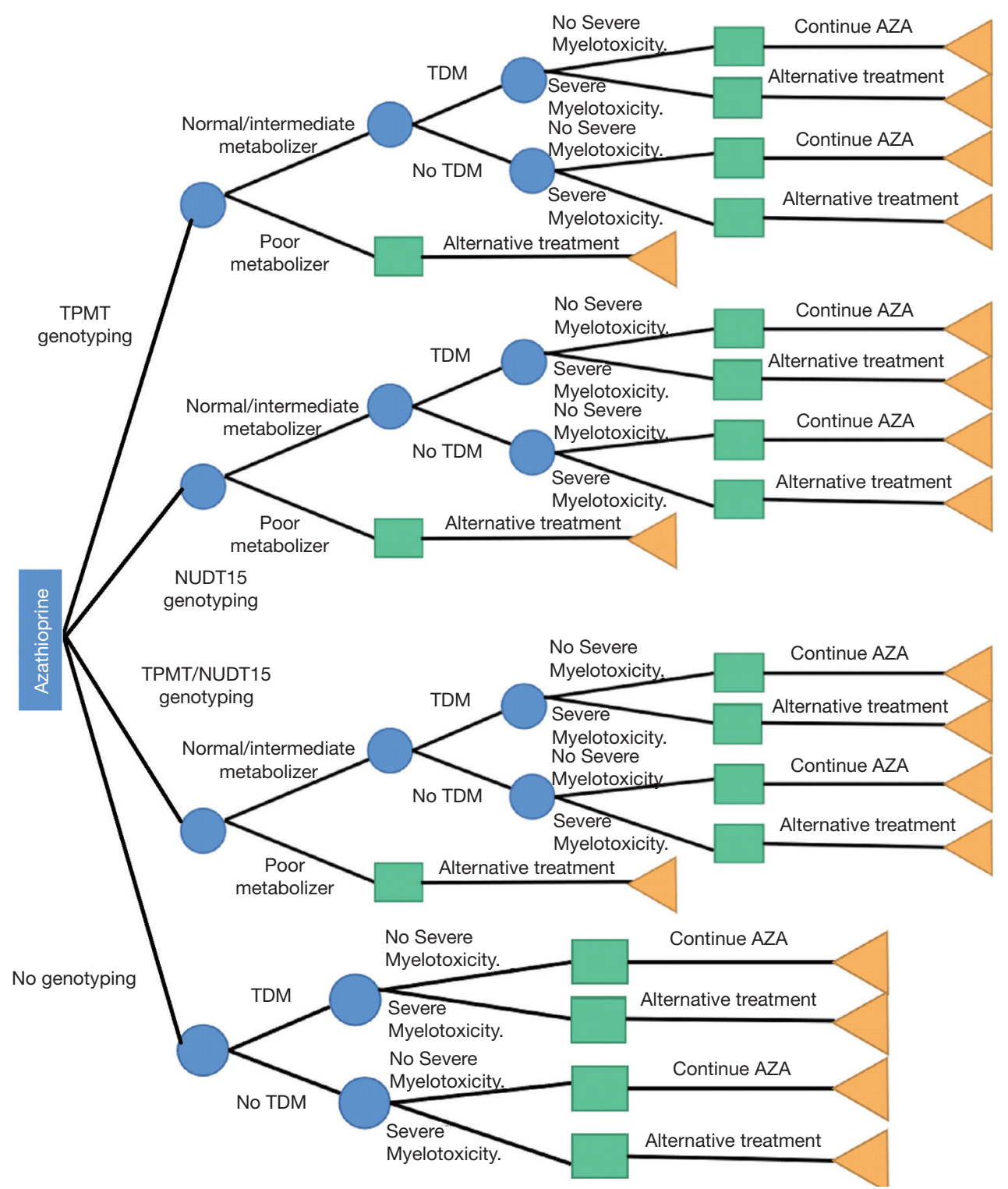

Figure 1 Decision tree of the baseline case. Circles represent chance nodes and squares represent a decision node.

TPMT/NUDT15 genotyping; and (IV) no genotyping. To evaluate the impact of TDM for AZA treatment, patients in the above 4 screening strategies were further subdivided according to whether or not TDM was performed. For the calculation of anticipated costs, the probabilities and costs of each branch were merged. A decision tree was designed to determine whether the genetic screening strategies were cost-effective (Figure 1). A decision tree was selected instead of other modeling methods, as the most straightforward method for decision-analytical modeling offered adequate representation for the different scenarios.

Based on the Clinical Pharmacogenomics Implementation
Consortium (CPIC) interpretation statement, the homozygous individuals were considered as poor metabolizers and included as an alternative treatment arm owing to their elevated risk of severe myelotoxicity (17). According to the American Gastroenterological Association Institute Guidelines, patients for whom severe myelotoxicity (poor metabolizers/leukopenia or pancytopenia needing hospital admission for treatment) was predicted in response to AZA treatment were transitioned to anti-tumor necrosis factor alpha (TNF- $\alpha)$ or methotrexate therapies. Patients who exhibited both negative TPMT and NUDT15 results (normal metabolizer) were given standard doses of AZA therapy, while 
Table 1 Demographic information of patients with IBD who were enrolled in the study

\begin{tabular}{lc}
\hline Variables & Value \\
\hline Total $(\mathrm{n})$ & 391 \\
Male, $\mathrm{n}(\%)$ & $265(67.8)$ \\
$\mathrm{BMI}\left(\mathrm{kg} / \mathrm{m}^{2}\right)$ & $19.7 \pm 3.5$ \\
$\mathrm{CD}, \mathrm{n}(\%)$ & $327(83.6)$ \\
$\mathrm{UC}, \mathrm{n}(\%)$ & $64(16.4)$ \\
Median age at start AZA (years) & $33.4 \pm 11.7$ \\
\hline
\end{tabular}

$\mathrm{IBD}$, inflammatory bowel disease; BMI, body mass index; $C D$, Crohn's disease; UC, ulcerative colitis; AZA, azathioprine.

those with intermediate enzymatic activity/heterozygous genotype (intermediate metabolizer) were given a $50 \%$ dose reduction.

\section{Patient population}

Data were obtained over 3 years (January 1, 2017 to October $1,2020)$ from an intercept cohort of patients based at the First Affiliated Hospital of Fujian Medical University, Fujian, China, all of whom had a diagnosis of IBD. The IBD database, which includes every patient to receive treatment for IBD in the hospital's Department of Gastroenterology (an IBD center), was established in 2017. IBD was diagnosed according to standard clinical, radiological, histological, and endoscopic evaluation results. All patients with IBD who received AZA between January 1, 2017 and October 1, 2020 underwent examination for therapeutic effectiveness and toxicity of AZA. Pregnant women and children were excluded.

Table 1 lists the demographics of the 391 enrolled IBD patients. Males accounted for 265 (67.8\%) of all study participants. The median body mass index (BMI) of the study participants was $19.7 \mathrm{~kg} / \mathrm{m}^{2}$ (range, $16.2-23.2 \mathrm{~kg} / \mathrm{m}^{2}$ ). In total, 327 (83.6\%) and 64 (16.4\%) patients had a diagnosis of Crohn's disease and ulcerative colitis, respectively (Table 1). At thiopurine therapy initiation, the patients had a median age of 33.4 years (range, 21.7-45.1 years).

\section{Model inputs}

\section{Probability parameters}

Based on retrospective data collected from the First Affiliated Hospital of Fujian Medical University, the efficacy of genotype screening strategies and TDM in predicting severe myelotoxicity induced by AZA in patients with/ without TPMT/NUDT15 deficiency were evaluated. The probability of severe myelotoxicity (absolute neutrophil count $<0.5 \times 10^{9} / \mathrm{L}$ or pancytopenia needing hospital admission for treatment) (18) was set based on retrospective data for base-case analysis. We set the prevalence of TPMT/ NUDT15 deficiency according to retrospective data for the base-case analysis, and ethnicity based on publicly available genomic data and previous literatures $(14,15,19-22)$ for sensitivity analyses (Table 2).

\section{Costs and utilities}

Costs were extracted from retrospective data collected at the First Affiliated Hospital of Fujian Medical University. All costs were expressed in US dollars (2020 annual average exchange rate) and undiscounted. The conversion of Chinese yuan (CNY) to US dollars was based on a 2020 average exchange rate of $6.89 \mathrm{CNY}=1.00 \mathrm{US}$ dollar (23). TPMT or NUDT15 genotyping cost $\$ 51.5$, combined TPMT/ NUDT15 genotyping cost $\$ 103$, and TDM cost $\$ 42.9$ each time. The cost of standard-dose AZA, 50\%-reduceddose AZA, and methotrexate, including prescription and laboratory test fees (i.e., complete blood count and blood chemistry for liver enzymes), was $\$ 243, \$ 182$, and $\$ 211$ per year per patient, respectively, over follow-up lasting 1 year. The cost associated with TNF- $\alpha$ inhibitor use, including office visits and hospitalization for infusion, was approximated to be $\$ 20,457$ per year per patient.

Expenses associated with severe myelotoxicity, including the overall costs of blood and platelet transfusion, granulocyte colony-stimulation factor use, antibiotics, and fees for using the isolation room in the course of hospitalization, were also analyzed. Based on analysis of the expenses for 2017-2020 hospital stays for AZA-induced severe myelotoxicity, the lowest, median (base-case analysis), and most severe case costs were taken as $\$ 290, \$ 363$, and $\$ 436$, respectively.

Utility value for IBD remission was adopted from the model input values of a previously published costeffectiveness analysis (24). For severe myelotoxicity cases, the utility value was sourced from published articles (25). Table 3 lists comprehensive details of the costs and utility values.

\section{Cost-effectiveness analysis}

The primary outputs of the model were the total cost, 
Table 2 Frequencies of phenotypes and the probability of severe myelotoxicity

\begin{tabular}{|c|c|c|}
\hline Variable & Value & References \\
\hline Poor metabolizer by TPMT genotyping & 0.00037 & Real-world data \\
\hline Poor metabolizer by NUDT15 genotyping & 0.01600 & Real-world data \\
\hline Poor metabolizer by combined TPMT/NUDT15 genotyping & 0.00014 & Real-world data \\
\hline \multicolumn{3}{|l|}{ Normal/intermediate metabolizer by TPMT genotyping } \\
\hline With TDM & 0.00001 & Real-world data \\
\hline Without TDM & 0.04600 & Real-world data \\
\hline \multicolumn{3}{|l|}{ Normal/intermediate metabolizer by NUDT15 genotyping } \\
\hline \multicolumn{3}{|c|}{ Normal/intermediate metabolizer by combined TPMT/NUDT15 genotyping } \\
\hline With TDM & 0.00001 & Real-world data \\
\hline Without TDM & 0.01430 & Real-world data \\
\hline \multicolumn{3}{|l|}{ No genotyping } \\
\hline With TDM & 0.05300 & Real-world data \\
\hline Without TDM & 0.09100 & Real-world data \\
\hline
\end{tabular}

TPMT, thiopurine methyl transferase; NUDT15, nucleotide triphosphate diphosphatase; TDM, therapeutic drug monitoring.

quality-adjusted life-years (QALYs), and incremental costeffectiveness ratios (ICERs). The estimation of QALYs was made based on the time spent in each state weighted by the utility of each state. ICERs were obtained by dividing the cost difference among the 4 possible interventions by the difference among their QALYs. ICER not exceeding the willingness-to-pay (WTP) threshold of \$30,425/QALY was considered cost-effective. The WTP threshold was set to 3 times the 2019 per capita Chinese gross domestic product in accordance with the World Health Organization (WHO)'s recommendation for cost-effectiveness analysis (26).

\section{Sensitivity analyses}

To test the robustness of the model, we conducted a series of sensitivity analyses. In the one-way sensitivity analysis, all variables changed within a plausible range (derived from credible intervals or through the assumption of a $20 \%$ variance from base-case values) $(27,28)$. We performed 10,000 Monte Carlo simulations for conducting probabilistic sensitivity analysis, with the variables concurrently varied with a specific pattern of distribution as shown in Table 3.

\section{Results}

\section{Base-case analysis}

The model with a 1-year horizon was used to estimate treatment costs and outcomes, and the results are displayed in Table 4. The base-case analysis uncovered marginal differences among the 4 strategies with respect to their overall anticipated costs. For 1 year, the mean costs of the NUDT15 genotyping, combined TPMT/NUDT15 genotyping, TPMT genotyping, and no genotyping strategies were estimated to be $\$ 608.94$, $\$ 622.77$, \$647.92, and $\$ 721.82$ per patient, respectively. The strategy without any genotyping had the highest cost (\$721.82/patient), with the worst health outcome (0.87873 QALY); it was identified as an undominated strategy. Compared to NUDT15 genotyping, combined TPMT/NUDT15 genotyping cost an additional \$13.83, yielding an ICER of \$3929.54/QALY, which was less than the WTP of $\$ 30,425$ per QALY in 
Table 3 Sensitivity analysis of parameters' ranges and distributions

\begin{tabular}{|c|c|c|c|c|}
\hline Variable & Baseline value & Range & Distribution & References \\
\hline Cost of TPMT genotyping, $\$$ & 51.5 & $41.2-61.8$ & Gamma & Real-world data \\
\hline Cost of NUDT15 genotyping, \$ & 51.5 & $41.2-61.8$ & Gamma & Real-world data \\
\hline Cost of combined genotyping, $\$$ & 103 & $82.4-123.6$ & Gamma & Real-world data \\
\hline Cost of standard dose of AZA, \$/year & 243 & 194.9-291.6 & Gamma & Real-world data \\
\hline Cost of $50 \%$ dose reduction in $\mathrm{AZA}, \$ /$ year & 182 & $145.6-219.4$ & Gamma & Real-world data \\
\hline Cost of methotrexate, $\$ /$ year & 211 & $168.8-253.2$ & Gamma & Real-world data \\
\hline Cost of TNF- $\alpha$ inhibitors, $\$ /$ year & 20,457 & $16,365.5-24,548.4$ & Gamma & Real-world data \\
\hline Poor metabolizer by TPMT genotyping & 0.00037 & $0.0014-0.015$ & Beta & (19) \\
\hline Poor metabolizer by NUDT15 genotyping & 0.016 & $0.0016-0.030$ & Beta & $(20,21)$ \\
\hline Poor metabolizer by combined genotyping & 0.00014 & $0.00-0.00456$ & Beta & $(22)$ \\
\hline \multicolumn{5}{|l|}{ Utilities } \\
\hline IBD & 0.89 & $0.83-0.92$ & Beta & (24) \\
\hline IBD with severe myelotoxicity & 0.66 & $0.6-0.68$ & Beta & (25) \\
\hline
\end{tabular}

TPMT, thiopurine methyl transferase; NUDT15, nucleotide triphosphate diphosphatase; TDM, therapeutic drug monitoring; AZA, azathioprine; IBD, inflammatory bowel disease.

Table 4 Outcomes in the base-case analysis

\begin{tabular}{lccc}
\hline Strategy & $\begin{array}{c}\text { Mean cost, } \\
\$\end{array}$ & $\begin{array}{c}\text { Total } \\
\text { QALYs }\end{array}$ & ICER $^{\mathrm{a}}$ \\
\hline NUDT15 genotyping & 608.94 & 0.88303 & $3,929.54$ \\
TPMT/NUDT15 genotyping & 622.77 & 0.88655 & NA \\
TPMT genotyping & 647.92 & 0.88249 & $-6,196.29$ \\
No genotyping & 721.82 & 0.87873 & $-12,659.62$ \\
\hline
\end{tabular}

a, ICER compared to TPMT/NUDT15 genotyping. TPMT, thiopurine methyl transferase; NUDT15, nucleotide triphosphate diphosphatase; NA, not applicable; QALYs, quality-adjusted life-years; ICER, incremental cost-effectiveness ratio.

China. Compared to the strategies with TPMT genotyping alone and no genotyping, the combined TPMT/NUDT15 genotyping strategy gained 0.00406 and 0.00782 QALYs and reduced the cost by $\$ 25.15$ and $\$ 99.06$, respectively, suggesting that the combination of TPMT and NUDT15 genotyping would be a cost-saving strategy.
The results of strategies with/without TDM are summarized in Table 5. Incorporating TDM for AZA treatment management proved to be more effective and less costly than not incorporating TDM, especially for the strategy with no genotyping. No genotyping with TDM had a lower cost and gained more QALYs than no genotyping without TDM, which indicated that the application of TDM in IBD management can still be costeffective in the absence of genotype screening technology.

\section{Sensitivity analyses}

\section{One-way sensitivity analysis}

To compare the relative importance of the parameters affecting ICERs, one-way sensitivity analysis was carried out. Because combined genotyping had been shown to dominate TPMT genotyping alone and no genotyping in the earlier analysis, only the ICERs of combined TPMT/ NUDT15 genotyping and NUDT15 genotyping were included in the tornado diagram (Figure 2). We found that 
Table 5 Results of strategies with and without TDM

\begin{tabular}{|c|c|c|c|}
\hline Strategy & Mean cost, \$ & Total QALYs & ICER $^{a}$ \\
\hline \multicolumn{4}{|c|}{ NUDT15 genotyping } \\
\hline With TDM & 321.24 & 0.88625 & NA \\
\hline Without TDM & 669.16 & 0.88226 & $-87,197.99$ \\
\hline \multicolumn{4}{|c|}{ TPMT/NUDT15 genotyping } \\
\hline With TDM & 331.06 & 0.88762 & NA \\
\hline Without TDM & 630.03 & 0.88521 & $-124,053.94$ \\
\hline \multicolumn{4}{|c|}{ TPMT genotyping } \\
\hline With TDM & 335.75 & 0.88301 & NA \\
\hline Without TDM & 762.82 & 0.88024 & $-154,176.89$ \\
\hline \multicolumn{4}{|l|}{ No genotyping } \\
\hline With TDM & 225.17 & 0.88502 & NA \\
\hline Without TDM & $1,146.23$ & 0.87340 & $-79,265.06$ \\
\hline
\end{tabular}

the ICERs depended highly on the costs associated with severe myelotoxicity, TPMT genotyping, and TDM testing. Other variables, including the utility for IBD and utility for IBD with severe myelotoxicity only moderately or marginally influenced the ICER. Each variable led to an ICER value under the Chinese WTP threshold. Together, these results served as confirmation of the model's robustness.

\section{Probabilistic sensitivity analysis}

The probability of the diagnostic tests being cost-effective was depicted through the generation of cost-effectiveness acceptability curves. At the WTP threshold of $\$ 30,425$ per QALY, combined TPMT/NUDT15 genotyping was determined to have a higher probability of being costeffective than NUDT15 genotyping (Figure 3), with a 91.7\% probability.

\section{Discussion}

Because of the massive demand for IBD treatment and the growing emphasis on economic evaluations of healthcare strategies, the present study was conducted in an effort to precisely assess the cost-effectiveness of genotype screening and TDM for IBD patients undergoing AZA therapy for the first time. By stratifying study enrollees on the basis of whether or not TDM was used, we revealed that the combination of TPMT/NUDT15 genotyping with TDM for IBD patients treated with AZA is likely to be optimal at the Chinese WTP threshold of $\$ 30,425$ per QALY. The sensitivity analysis further showed a higher than $90 \%$ probability that TPMT/NUDT15 genotyping of IBD patients treated with AZA was cost-effective, due to the avoidance of episodes of severe myelosuppression and the net gain of health benefits compared with other genotyping strategies. Combined TPMT/NUDT15 genotyping to avoid AZA-related severe myelotoxicities was a key factor propelling economic outcomes. Moreover, the base-case analysis uncovered marginal differences between the TPMT genotyping strategy and no genotyping strategy with respect to their overall utilities, and the mean difference was 0.00376 QALYs. TPMT genotype-guided thiopurine treatment in IBD patients reduced the risk of ADR among patients carrying a TPMT variant, without increasing overall healthcare costs and resulting in comparable quality of life, as compared to no genotype-guided treatment. Our one-way sensitivity analysis of combined TPMT/NUDT15 genotyping versus NUDT15 genotyping uncovered the cost associated with severe myelotoxicity to have the greatest influence of any model input.

Since a significant proportion of adverse events is caused by genetic differences in individual treatment responses, the findings of this study suggest that identifying the risk of myelotoxicity through genotypic screening before the initiation of AZA, together with the inclusion of TDM in the management of AZA treatment, would improve adverse reactions and provide considerable medical and economic benefits. Despite the TPMT mutation rate in Asians being lower than that in Caucasians, the incidence of leukopenia is as high as $40 \%$ in Asians (29). Notably, deficiency of the NUDT15 gene represents a major genetic determinant in patients of Asian origin (20). Due to the rare reporting of NUDT15 risk variants in European and African populations, NUDT15-guided thiopurine dosing in these populations is thought to be limited. Despite this, genetic testing of NUDT15 could be of clinical use for identifying Asian patients who have an elevated risk of thiopurineinduced leukopenia. Thus, combined TPMT/NUDT15 genotyping is presumed to have a significant impact on reducing AZA-related severe myelotoxicities (14).

The present study has evidenced combined TPMT/ NUDT15 genotyping to be a cost-effective strategy for patients with IBD, which can help to avoid severe myelosuppression associated with AZA therapy from the 


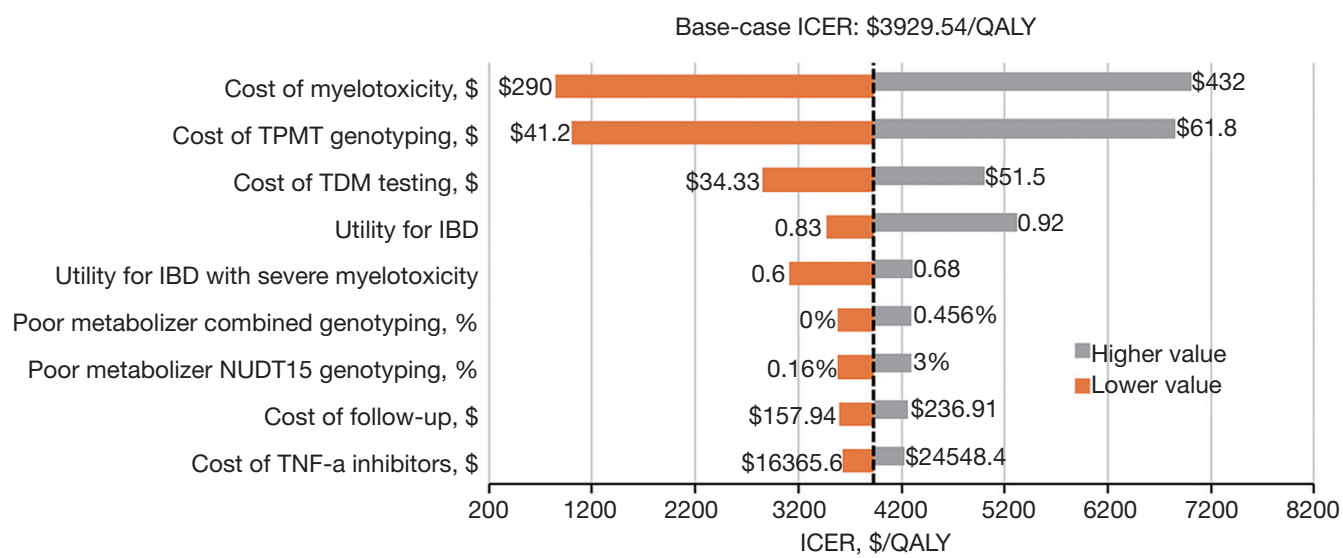

Figure 2 Results of one-way sensitivity analysis of combined TPMT/NUDT15 genotyping versus NUDT15 genotyping. The diagram compares the incremental cost-effectiveness ratios (ICERs) of combined TPMT/NUDT15 genotyping and NUDT15 genotyping for different model input parameters. The model's robustness was tested by performing one-way sensitivity analysis by varying key parameters over plausible ranges to assess their global effect on the ICER. The longer the bar, the greater the sensitivity of the global results to variations in that key parameter. The orange bar represents the ICER at the minimum parameter value, and the gray bar represents the ICER at the maximum parameter value. ICER, incremental cost-effectiveness ratio; QALYs, quality-adjusted life-years; TPMT, thiopurine methyl transferase; NUDT15, nucleotide triphosphate diphosphatase; TDM, therapeutic drug monitoring.

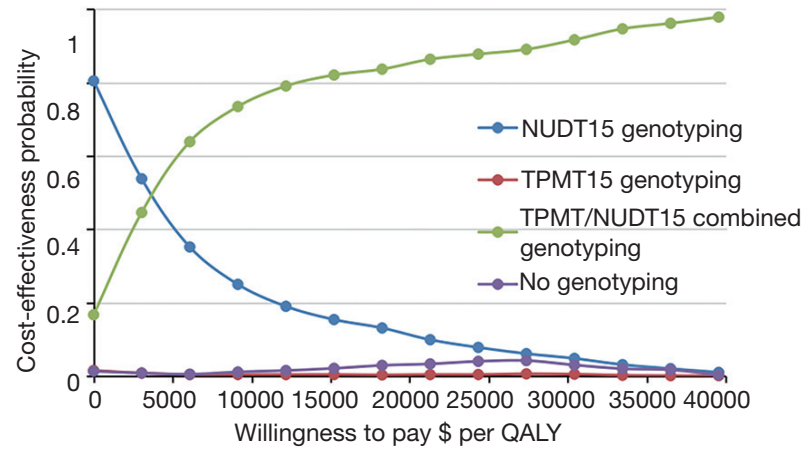

Figure 3 Cost-effectiveness acceptability curves. Probabilistic sensitivity analyses results on the basis of 10,000 Monte Carlo simulations, which includes the sampling of model variable values from distributions imposed on variables to indicate uncertainty regarding the cost-effectiveness of combined genotyping at various willingness-to-pay thresholds. The combined genotyping strategy has a $91.7 \%$ chance of being cost-effective at a threshold of $\$ 30,425$ per QALY in China. TPMT, thiopurine methyl transferase; NUDT15, nucleotide triphosphate diphosphatase; QALYs, quality-adjusted life-years.

perspective of the Chinese healthcare system. This result is consistent with previously published literature reviews of pharmacogenomics-guided treatment. A study conducted by Zarca et al. (14) demonstrated that combination screening for NUDT15 and TPMT defective alleles shows costeffectiveness in the treatment of patients of Asian descent; however, the opposite conclusion was reached for Caucasian populations due to the very low frequency of NUDT15 functional allelic variants. Winter et al. (30) reported that pretreatment screening for TPMT polymorphisms demonstrated good value in patients with IBD beginning AZA treatment. Similarly, Yao et al. (1) described TDMguided strategies for IBD management as being costeffective.

The strengths of our study included a comprehensive analysis, which synthesized real-world data and epidemiological evidence through an economic modeling approach in the context of the Chinese healthcare system. This analysis also simultaneously assessed the costeffectiveness of combined TPMT/NUDT15 genotyping and TDM for AZA treatment management. The information provided by our economic analysis will be helpful for physicians, patients as well as other decision makers. The utilization of this cost-effective strategy may help to improve the tolerability of AZA regimens for at-risk patients with IBD in the future, lowering the rate of ADRs and the associated costs for the Chinese national healthcare system in the future.

The following considerations and limitations should be considered when interpreting the results of this cost- 
effectiveness study. Firstly, important data on patients' quality of life were not included in the hospital's IBD database; thus, we sourced this information from previously published papers (22). Nonetheless, we conducted a sensitivity analysis on the utility values, and the oneway analysis revealed that they were not the key factors influencing the results, having minimal impact. Additionally, the estimates of the severe myelosuppression incidence and costs were obtained from a local hospital due to the lack of published data in China. The First Affiliated Hospital of Fujian Medical University is among the best-equipped large-scale hospitals (top $1^{\text {st }}$-class) in the country; the hospital treats nearly all IBD patients in Fujian. In general, the cost of drugs makes up a considerable proportion of the overall cost for IBD treatment. Most drugs in the same geographical area (province) cost the same. Because of economic disparities in China, unit costs of hospitals may vary from province to province; however, this issue was addressed by the sensitivity analyses. Finally, studies shown that assessment of TPMT enzyme activity is superior to genotype in predicting myelosuppression following AZA therapy in patients with IBD (31). In addition, Zarca et al. (18) demonstrated that the phenotype-based strategy of screening for TPMT deficiency dominates (cheaper and more effective) the genotype-based screening strategy in France. Because of the differences in the national conditions and medical environments, this finding cannot be extrapolated to patients with IBD in China. Moreover, due to lack of the data regarding phenotyping-based dosing of TPMT activity, the cost-effectiveness of phenotypingbased dosing of TPMT activity cannot be accessed for the time being. If the data of TPMT enzyme activity in the real-world in China are collected in the future, the results updates of our study might be warranted correspondingly.

Colitis-associated colorectal cancer (CAC) is one of the most severe complications of IBD and constitutes the cause of death in $10-15 \%$ of patients. The molecular mechanism of CAC has not been fully investigated; previous study demonstrated that inflammatory responses lead to mutations and epigenetic changes in normal cells. Reactive oxygen species produced by myeloid cells induce mutations in intestinal epithelial cells (32). In addition, inflammatory responses also contribute to creating transformed and malignant clones. Therefore, controlling intestinal inflammation is a key preventive and therapeutic strategy for CAC. Furthermore, studies indicated that the key steps for complete mucosal healing in IBD are anti-inflammatory and proresolution therapy. However, it is pertinent to note that there are surprisingly no current bona fide proresolution or repair treatments available to patients with IBD (33). Because the mechanism and key driving factors that initiate and perpetuate IBD mucosal inflammation are not fully known, the dominant drug development model so far has been based on the principle of long-term continuous inhibition of the abnormal immune response in IBD. Presumably, it is difficult to find an approach by combining different biologics or using biomarkers to select treatment. If definite biomarkers were confirmed, it will certainly be helpful for treatment. It is necessary to comprehensively evaluate and flexibly formulate IBD treatment strategies based on different severity, extent of disease, treatment response, and different outcomes and prognosis. Based on the treatment guidelines, for IBD patients at high risk of disease progression, the top-down treatment (early application of biotherapy) may be the best strategy to achieve the best therapeutic effect.

To conclude, this study has demonstrated that a strategy combining genotypic testing of NUDT15 and TPMT prior to the initiation of AZA treatment and incorporating TDM into AZA treatment management has good costeffectiveness for Chinese patients with IBD.

\section{Acknowledgments}

Funding: The study was supported by the Social Development Guiding (Key) Project of Fujian Provincial Science and Technology Department (2020Y0027), Pinfang Huang.

\section{Footnote}

Reporting Checklist: The authors have completed the CHEERS reporting checklist. Available at https://dx.doi. org/10.21037/atm-21-1980

Data Sharing Statement: Available at https://dx.doi. org/10.21037/atm-21-1980

Conflicts of Interest: All authors have completed the ICMJE uniform disclosure form (available at https://dx.doi. org/10.21037/atm-21-1980). Prof. PH reports that this study was supported by the Social Development Guiding (Key) Project of Fujian Provincial Science and Technology Department, 2020Y0027. The other authors have no conflicts of interest to declare.

Ethical Statement: The authors are accountable for all 
aspects of the work in ensuring that questions related to the accuracy or integrity of any part of the work are appropriately investigated and resolved. All procedures performed in this study involving human participants were in accordance with the Declaration of Helsinki (as revised in 2013). The study was approved by the Ethics Committee of the First Affiliated Hospital of Fujian Medical University (approval number: 2015-131). All patients provided written informed consent before participation.

Open Access Statement: This is an Open Access article distributed in accordance with the Creative Commons Attribution-NonCommercial-NoDerivs 4.0 International License (CC BY-NC-ND 4.0), which permits the noncommercial replication and distribution of the article with the strict proviso that no changes or edits are made and the original work is properly cited (including links to both the formal publication through the relevant DOI and the license). See: https://creativecommons.org/licenses/by-nc-nd/4.0/.

\section{References}

1. Yao J, Jiang X, You JHS. A Systematic Review on Costeffectiveness Analyses of Therapeutic Drug Monitoring for Patients with Inflammatory Bowel Disease: From Immunosuppressive to Anti-TNF Therapy. Inflamm Bowel Dis 2021;27:275-82.

2. Adam L, Phulukdaree A, Soma P. Effective long-term solution to therapeutic remission in Inflammatory Bowel Disease: Role of Azathioprine. Biomed Pharmacother 2018;100:8-14.

3. Sluiter RL, Van Marrewijk C, De Jong D, et al. GenotypeGuided Thiopurine Dosing Does not Lead to Additional Costs in Patients With Inflammatory Bowel Disease. J Crohns Colitis 2019;13:838-45.

4. Mushtaq K, Khan Z, Aziz M, et al. Trends and outcomes of fungal infections in hospitalized patients of inflammatory bowel disease: a nationwide analysis. Transl Gastroenterol Hepatol 2020;5:35.

5. McLeod HL, Krynetski EY, Relling MV, et al. Genetic polymorphism of thiopurine methyltransferase and its clinical relevance for childhood acute lymphoblastic leukemia. Leukemia 2000;14:567-72.

6. Relling MV, Gardner EE, Sandborn WJ, et al. Clinical pharmacogenetics implementation consortium guidelines for thiopurine methyltransferase genotype and thiopurine dosing: 2013 update. Clin Pharmacol Ther 2013;93:324-5.

7. Tumer TB, Ulusoy G, Adali O, et al. The low frequency of defective TPMT alleles in Turkish population: a study on pediatric patients with acute lymphoblastic leukemia. Am J Hematol 2007;82:906-10.

8. Walker GJ, Harrison JW, Heap GA et al. Association of Genetic Variants in NUDT15 With Thiopurine-Induced Myelosuppression in Patients With Inflammatory Bowel Disease. JAMA 2019;321:773-85.

9. Moriyama T, Nishii R, Perez-Andreu V, et al. NUDT15 polymorphisms alter thiopurine metabolism and hematopoietic toxicity. Nat Genet 2016;48:367-73.

10. Cargnin S, Genazzani AA, Canonico PL, et al. Diagnostic accuracy of NUDT15 gene variants for thiopurineinduced leukopenia: a systematic review and meta-analysis. Pharmacol Res 2018;135:102-11.

11. Papamichael K, Cheifetz AS, Melmed GY, et al. Appropriate Therapeutic Drug Monitoring of Biologic Agents for Patients With Inflammatory Bowel Diseases. Clin Gastroenterol Hepatol 2019;17:1655-68.e3.

12. Desai R, Patel U, Goyal H, et al. In-hospital outcomes of inflammatory bowel disease in cannabis users: a nationwide propensity-matched analysis in the United States. Ann Transl Med 2019;7:252.

13. Husereau D, Drummond M, Petrou S et al. Consolidated Health Economic Evaluation Reporting Standards (CHEERS) statement. BMJ 2013;346:f1049.

14. Zarca K, Chansavang A, Loriot MA, et al. Costeffectiveness analysis of pretreatment screening for NUDT15 defective alleles. Pharmacogenetics and Genomics 2020;30:175-83.

15. Iacucci $M, M c Q u a i d ~ K$, Gui X, et al. A multimodal (FACILE) classification for optical diagnosis of inflammatory bowel disease associated neoplasia. Endoscopy 2019;51:133-41.

16. Connell WR, Kamm MA, Ritchie JK, et al. Bone marrow toxicity caused by azathioprine in inflammatory bowel disease: 27 years of experience. Gut 1993;34:1081-5.

17. Relling MV, Schwab M, Whirl-Carrillo M, et al. Clinical Pharmacogenetics Implementation Consortium Guideline for Thiopurine Dosing Based on TPMT and NUDT15 Genotypes: 2018 Update. Clin Pharmacol Ther 2019;105:1095-105.

18. Zarca K, Durand-Zaleski I, Loriot MA, et al. Modeling the Outcome of Systematic TPMT Genotyping or Phenotyping Before Azathioprine Prescription: A CostEffectiveness Analysis. Mol Diagn Ther 2019;23:429-38.

19. Wang HH, He Y, Wang HX, et al. Comparison of TPMT and NUDT15 polymorphisms in Chinese patients with inflammatory bowel disease. World J Gastroenterol 
2018;24:941-8.

20. Provenzale D, Onken JE. TPMT genotype screening for patients about to begin azathioprine treatment--a look at costs and potential benefits. Inflamm Bowel Dis 2005;11:1119-20.

21. Yang JJ, Landier $W$, Yang $W$ et al. Inherited NUDT15 Variant Is a Genetic Determinant of Mercaptopurine Intolerance in Children With Acute Lymphoblastic Leukemia. J Clin Oncol 2015;33:1235-42.

22. Sutiman N, Chen S, Ling KL et al. Predictive role of NUDT15 variants on thiopurine-induced myelotoxicity in Asian inflammatory bowel disease patients. Pharmacogenomics 2018;19:31-43.

23. Available online: http://www.exchangerates24.com/

24. You JHS, Jiang X, Lee WH, et al. Cost-effectiveness analysis of fecal microbiota transplantation for recurrent Clostridium difficile infection in patients with inflammatory bowel disease. J Gastroenterol Hepatol 2020;35:1515-23.

25. Thompson AJ, Newman WG, Elliott RA, et al. The CostEffectiveness of a Pharmacogenetic Test: A Trial-Based Evaluation of TPMT Genotyping for Azathioprine. Value in Health 2014;17:22-33.

26. National Bureau of Statistics of China $>>$ Annual Data. Available online: http://www.stats.gov.cn/english/ Statisticaldata/AnnualData/

27. Wu B, Gu X, Zhang Q, et al. Cost-Effectiveness of Osimertinib in Treating Newly Diagnosed, Advanced

Cite this article as: Zeng D, Huang $X$, Lin S, Lin R, Weng X, Huang P. Cost-effectiveness analysis of genotype screening and therapeutic drug monitoring in patients with inflammatory bowel disease treated with azathioprine therapy: a Chinese healthcare perspective using real-world data. Ann Transl Med 2021;9(14):1138. doi: 10.21037/atm-21-1980
EGFR-Mutation-Positive Non-Small Cell Lung Cancer. Oncologist 2019;24:349-57.

28. Liubao P, Xiaomin W, Chongqing T et al. CostEffectiveness Analysis of Adjuvant Therapy for Operable Breast Cancer from a Chinese Perspective: Doxorubicin plus Cyclophosphamide versus Docetaxel plus Cyclophosphamide. PharmacoEconomics 2009; 27:873-86.

29. Lee KM, Kim YS, Seo GS et al. Use of Thiopurines in Inflammatory Bowel Disease: A Consensus Statement by the Korean Association for the Study of Intestinal Diseases (KASID). Intest Res 2015;13:193.

30. Winter J, Walker A, Shapiro D, et al. Cost-effectiveness of thiopurine methyltransferase genotype screening in patients about to commence azathioprine therapy for treatment of inflammatory bowel disease. Aliment Pharmacol Ther 2004;20:593-9.

31. Winter JW, Gaffney D, Shapiro D, et al. Assessment of thiopurine methyltransferase enzyme activity is superior to genotype in predicting myelosuppression following azathioprine therapy in patients with inflammatory bowel disease. Aliment Pharmacol Ther 2007;25:1069-77.

32. Greten FR, Grivennikov SI. Inflammation and Cancer: Triggers, Mechanisms, and Consequences. Immunity 2019;51:27-41.

33. Ho GT, Cartwright JA, Thompson EJ, et al. Resolution of Inflammation and Gut Repair in IBD: Translational Steps Towards Complete Mucosal Healing. Inflamm Bowel Dis 2020;26:1131-43. 